\begin{tabular}{c|c|c|}
\hline \hline & MARINE ECOLOGY PROGRESS SERIES \\
Vol. 273: 291-302, 2004 & Mar Ecol Prog Ser & Published June 8 \\
\hline \hline
\end{tabular}

\title{
Network structure and robustness of marine food webs
}

\author{
Jennifer A. Dunne ${ }^{1,4, *}$, Richard J. Williams ${ }^{2,4}$, Neo D. Martinez ${ }^{3,4}$ \\ ${ }^{1}$ Santa Fe Institute, 1399 Hyde Park Road, Santa Fe, New Mexico 87501, USA \\ ${ }^{2}$ National Center for Ecological Analysis and Synthesis, University of California Santa Barbara, 735 State St., Suite 300, \\ Santa Barbara, California 93101-3351, USA \\ ${ }^{3}$ Center for Applied Mathematics, 657 Frank HT Rhodes Hall, Cornell University, Ithaca, New York 14853, USA \\ ${ }^{4}$ Pacific Ecoinformatics and Computational Ecology Lab, Rocky Mountain Biological Laboratory, PO Box 519, Crested Butte, \\ Colorado 81224, USA
}

\begin{abstract}
Previous studies suggest that food-web theory has yet to account for major differences in food-web properties of marine versus other types of ecosystems. We examined this issue by analyzing the network structure of food webs for the Northeast US Shelf, a Caribbean reef, and Benguela, off South Africa. The values of connectance (links per species ${ }^{2}$ ), link density (links per species), mean chain length, and fractions of intermediate, omnivorous, and cannibalistic taxa of these marine webs are somewhat high but still within the ranges observed in other webs. We further compared the marine webs by using the empirically corroborated 'niche model' that accounts for observed variation in diversity (taxon number) and complexity (connectance). Our results substantiate previously reported results for estuarine, fresh-water, and terrestrial datasets, which suggests that food webs from different types of ecosystems with variable diversity and complexity share fundamental structural and ordering characteristics. Analyses of potential secondary extinctions resulting from species loss show that the structural robustness of marine food webs is also consistent with trends from other food webs. As expected, given their relatively high connectance, marine food webs appear fairly robust to loss of most-connected taxa as well as random taxa. Still, the short average path length between marine taxa (1.6 links) suggests that effects from perturbations, such as overfishing, can be transmitted more widely throughout marine ecosystems than previously appreciated.
\end{abstract}

KEY WORDS: Food webs - Network structure - Marine ecosystems $\cdot$ Robustness Connectance Niche model $\cdot$ Biodiversity loss

\section{INTRODUCTION}

Recent studies have described general properties of complex food-web structure as well as simple rules that accurately predict such structure in a wide variety of terrestrial and aquatic ecosystems (e.g. Williams \& Martinez 2000, Camacho et al. 2002a,b, Dunne et al. 2002a,b, Williams et al. 2002, Krause et al. 2003). The data used in these studies generally have substantially higher diversity and more comprehensive and even resolution of taxa at different trophic levels than the earlier 'ECOWeB' food-web data used to initiate topological food-web research (Briand \& Cohen 1984,
Cohen et al. 1990), which are still occasionally used today (Neutel et al. 2002). Analyses of improved data have modified earlier food-web theory and contributed new insights into the trophic network structure and robustness of ecosystems (Martinez 1991, Williams \& Martinez 2000, Dunne et al. 2002a,b). However, food webs from marine ecosystems have been conspicuously absent from recent synthetic work. An earlier analysis of ECOWeB data, including 11 marine food webs with low average diversity (18 trophic species), reported strong contrasts between marine and other ecosystems, with marine food webs having greater average links per species $(L / S)$ and chain lengths than 
other food webs (Cohen 1994). Similarly, a recent analysis of a more diverse, highly resolved marine food web (81 taxa) for the Northeast US Shelf found that $L / S$ and connectivity are either an order of magnitude higher than non-marine webs or are disproportionate to the diversity of the system (Link 2002). The study concluded, 'it is clear that the emergent properties of this marine food web are very different than their terrestrial and freshwater counterparts.'

Previous studies of marine food-web structure rely on making direct comparisons of food-web properties among marine systems and between marine and nonmarine systems. However, it is now well documented that most food-web properties are scale-dependent, meaning that they change as diversity and complexity change (e.g. Martinez 1993, 1994). This scaledependence makes direct comparison of properties among food webs with different levels of species richness and trophic interaction richness (which we use to refer collectively to measures such as links per species, connectivity, and connectance) potentially misleading. Comparing observed food webs against food webs generated by a simple model that accounts for different levels of ecological diversity and complexity provides one solution to this problem. This type of modeling approach is useful for at least 5 reasons. First, if a model successfully characterizes the overall network structure of a range of food webs, those food webs can be considered to have a similar topology. Second, a model can act as a benchmark for comparing observed food webs. The ways in which the structure of observed food webs varies from structure predicted by a model that incorporates scale-dependence provides a way to compare food webs with different diversity and complexity. Third, a model can help to identify problems with particular datasets and to quantify how methodological biases ramify through analysis. Fourth, a model that successfully characterizes the network structure of observed food webs can be used to extend our understanding of what food webs with even greater diversity or complexity than currently available might look like, and also how food-web properties may be expected to vary with those factors (e.g. Williams et al. 2002). Fifth, if a model is phenomenologically successful (i.e. fits current datasets well, and successfully predicts new datasets), its underlying assumptions can point us towards potential mechanisms that generate general patterns of food-web structure.

We focus on the first 3 issues using 2 food-web structure models: the cascade model (Cohen et al. 1990) and the niche model (Williams \& Martinez 2000). These simple models use only 2 input parameters: $S$ (species richness) and $C$ (connectance-links per species ${ }^{2}$, $L / S^{2}$ ). These parameters are set equal to the $S$ and $C$ of each empirical food web, and then multiple stochastic models of each food web are produced using simple ordering rules. The use of those 2 parameters embodies the notion that the network structure of food webs will vary in predictable ways in accordance with their diversity (i.e. number of taxa) and complexity (i.e. connectance).

We analyzed 4 recently published marine food webs with 29 to 249 taxa, representing 3 marine ecosystems. In particular, we considered the questions: (1) Do marine food webs show similarities to each other? (2) Do marine food webs look like other types of food webs? (3) Do food-web theory and models apply to marine ecosystems? (4) What are the empirical limitations of recent marine food-web data? We also used the marine datasets to simulate the potential effects of different types and magnitudes of species loss in triggering cascading secondary extinctions. This allows a comparison of the potential robustness to biodiversity loss associated with the network structure of marine versus other types of food webs (Dunne et al. 2002a). This type of research on food-web structure and robustness is just one example of the statistical mechanics of technology, information, social, and biotic networks (see Strogatz 2001, Albert \& Barabási 2002 for reviews). Even in the absence of dynamical modeling, which is challenging even for relatively low diversity systems, analysis of network structure can have important implications for network function (Strogatz 2001). For marine ecosystems, historically subject to intense fisheries pressure and subsequent collapse (Jackson et al. 2001, Pauly et al. 1998, 2002), more detailed knowledge of the complex network of trophic relationships that encompass species of economic interest will be important for guiding more sustainable policy.

\section{METHODS}

We analyzed the network structure of food webs with relatively detailed species and trophic interaction data from 3 marine ecosystems. The first, the Benguela ecosystem off the southwest coast of South Africa (Yodzis 1998, 2000), is represented by a 29-taxa food web. The second, the Northeast US Shelf ecosystem, is represented by an 81-taxa food web (Link 2002). The third, a Caribbean coral reef ecosystem from the Puerto RicoVirgin Islands shelf complex (Opitz 1996), is represented by 2 versions of the same food web, with 249 and 50 taxa. The original investigator generated the smaller food web from the larger dataset in order to analyze the smaller web with the ECOPATH II box model, which allows for a maximum of 50 compartments (Christensen \& Pauly 1992). For the smaller food web, 
243 fish species were aggregated into 25 fish groups based on size, activity level, and food type, while 41 non-fish taxa, many of which were already aggregated, were reduced further into 25 groups based on mortality, food consumption, size, diet composition, lifestyle, and taxonomic closeness (Opitz 1996). We refer to these ecosystems and their food webs as Benguela, NE US Shelf, and small and large Caribbean Reef.

We use the term 'taxa' to refer to the groups of organisms identified by the original investigators as the core units of analysis in their food webs. These range from species (e.g. spotted hake, humans), to species grouped by trophic habit, taxonomy, or other criteria (e.g. benthic filter feeders, macrozooplankton, whales and dolphins), to mixed pools (e.g. detritus - a combination of live organisms, organic matter, and inorganic matter). All 3 ecosystems are subject to intense human fishing, and the marine food-web data reflect a strong fish bias, which represent $\geq 50 \%$ of the taxa in each web (Table 1). The large version of the Caribbean Reef food web is particularly biased, with fish representing $84 \%$ of taxa. In all 4 webs, invertebrates (14 to $41 \%$ of taxa) are under-represented and poorly resolved relative to the fishes (as also noted by Link 2002) and basal taxa are very highly aggregated into groups such as 'phytoplankton' and 'detritus' (2 to $7 \%$ of taxa).

How does the quality of these datasets compare to earlier marine food webs used for structural studies, in particular the marine food webs from the $\mathrm{ECOWeB}$ database (Cohen et al. 1990, Cohen 1994) A 1993 article on 'Improving food webs' (Cohen et al. 1993) called for 'more explicitness and more exhaustiveness' in the compilation of food webs, and suggested the admittedly ideal goal that all species and all links between them for a particular ecological time and volume should be reported. However, this type of exhaustiveness is difficult or impossible (particularly when considering microorganisms and other cryptic organisms), may be unnecessary for certain kinds of investigations (Martinez et al. 1999), and may impede useful analytical and theoretical development focused on fundamental patterns and mechanisms. Instead, we suggest that, in addition to methodological explicitness (Cohen et al.

Table 1. Number (percentage) of different types of taxa in 4 marine food webs

\begin{tabular}{|lcccc|}
\hline & Benguela & $\begin{array}{l}\text { Caribbean } \\
\text { Reef (small) }\end{array}$ & $\begin{array}{l}\text { Caribbean } \\
\text { Reef (large) }\end{array}$ & $\begin{array}{c}\text { NE US } \\
\text { Shelf }\end{array}$ \\
\hline All taxa & 29 & 50 & 249 & 81 \\
Fishes & $18(62)$ & $25(50)$ & $208(84)$ & $41(51)$ \\
Other vertebrates & $3(10)$ & $2(4)$ & $2(1)$ & $5(6)$ \\
Invertebrates & $6(22)$ & $18(36)$ & $35(14)$ & $33(41)$ \\
Basal groups & $2(7)$ & $3(6)$ & $4(2)$ & $2(2)$ \\
\hline
\end{tabular}

1993), food web compilation should continually strive for higher degrees of consistency and comprehensiveness. The food webs in ECOWeB are generally plagued by low diversity, reflecting in many (but not all) cases a lack of comprehensiveness, as well as inconsistent resolution of different types of taxa, with lower, non-vertebrate trophic levels often highly aggregated. An example of one of the more diverse marine food webs in ECOWeB is web 29, a food web of the Arctic seas (Dunbar 1954). This web has 22 taxa, with 2 basal taxa, 4 invertebrate taxa, and 16 vertebrate taxa (primarily whales, seals, and fishes). While the Benguela, small Caribbean Reef, and NE US Shelf food webs similarly fail to distinguish among basal taxa, they are improved over the Arctic seas and other earlier marine food webs given their more comprehensive inclusion, higher resolution, and more even resolution of taxa at other trophic levels. The large Caribbean Reef web is particularly interesting: while it is an order of magnitude more diverse than earlier ECOWeB marine food webs, we do not consider it necessarily higher quality than many of the early food webs. The Arctic seas food web, despite its lack of diversity, is more consistently and evenly resolved than the large 249-taxa Caribbean Reef web with its $84 \%$ fish species and $<2 \%$ basal taxa. Because of the flaws of the large Caribbean Reef web, we limit most of our discussion of marine food-web characteristics to the Benguela, small Caribbean Reef, and NE US Shelf food webs.

We studied trophic species versions of the food webs. Trophic species are groups of taxa whose members share the same set of predators and prey (Briand \& Cohen 1984). The use of trophic species, hereafter referred to as species $(S)$, is a convention in structural food-web studies that can reduce methodological biases of uneven resolution of taxa within and among food webs (Briand \& Cohen 1984, Williams \& Martinez 2000). Trophic species aggregation alters the marine food webs very little, with no changes to the Benguela and small Caribbean Reef webs, and reductions of the NE US Shelf and large Caribbean food webs from 81 to 79 taxa and 249 to 245 taxa, respectively (see Table 2 ). The use of trophic species is not a panacea, as demonstrated by the large Caribbean Reef web. Although this food web is excessively biased towards fishes, the use of the trophic species aggregation does almost nothing to alleviate its extremely uneven resolution. The ecologically more sophisticated criteria used to aggregate the large food web into a smaller 50-taxa web (Opitz 1996) are much more effective than 'trophic species' in rendering a higher quality, more evenly resolved, albeit lower diversity, food web. 
For each food web, we calculated 18 network structure properties (see Tables $2 \& 3$ ). Two standard measures of food-web trophic interaction richness are reported: links per species $(L / S)$, which equals the mean number of species' predators plus prey, also referred to as link density; and connectance $(C)$, where $C=L / S^{2}$, the proportion of all possible trophic links $\left(S^{2}\right)$ that are actually realized $(L)$, also referred to as 'directed connectance.' Seven properties give percentages of types of species in a food web: top ( $T$ ) (taxa that lack any predators or parasites), intermediate $(I)$, and basal species $(B)$ (taxa that lack any prey items); cannibals (Can); omnivores (Omn) (taxa with food chains of different lengths, where a food chain is a linked path from a non-basal to a basal species); herbivores plus detritivores (Herb); and species involved in looping (Loop) by appearing in a food chain twice. Most of these are commonly calculated properties that have been reviewed elsewhere (e.g. Williams \& Martinez 2000), although Herb has not been previously reported.

The remaining 10 properties quantify overall properties of food-web network structure. We calculated a trophic level measure called the mean 'shortweighted trophic level' (TL) (Williams \& Martinez 2004). For a particular taxon, short-weighted trophic level is the average of 'prey-averaged trophic level' (1 plus the mean trophic level of all the taxon's trophic resources) and 'shortest trophic level' (1 plus the shortest chain length from the consumer taxon to a basal taxon). An examination of 6 ways of estimating trophic level based on network structure, compared to trophic level calculations based on more information-rich flow-weighted trophic data, shows that short-weighted trophic level gives the most accurate estimate of trophic level based on binary link information (Williams \& Martinez 2004). Shortweighted trophic level estimates, like estimates based on flow-weighted link information, produce lower trophic levels than those reported by studies using the more common 'chain-averaged trophic level' algorithm (Martinez 1991, Polis 1991, Fussman \& Heber 2002).

We report 3 food-chain-length related measures, the mean (ChLen) and standard deviation (ChSD) of chain lengths and the log of the number of chains (ChNum). We report the standard deviation of mean generality $(G e n S D)$, how many prey items a species has, and vulnerability ( $V u l S D$ ), how many predators a species has. These 2 measures quantify the variabilities of species' normalized predator and prey counts (Schoener 1989). The number of predators and prey shared in common by a pair of species divided by the pair's total number of predators and prey is referred to as trophic similarity. We averaged across each species' highest trophic similarity index to another species to report the mean maximum trophic similarity (MaxSim). The previous 6 properties follow Williams \& Martinez (2000). We also report 2 measures of 'small-world' network structure (Watts \& Strogatz 1998): characteristic path length (Path), the mean shortest path length between species pairs; and clustering coefficient (Clust), the mean fraction of species pairs connected to the same species that are connected to each other (Camacho et al. 2002b, Dunne et al. 2002b, Montoya \& Solé 2002, Williams et al. 2002).

For each marine food web, we compare the ability of 2 simple stochastic food-web structure models, the cascade model (Cohen et al. 1990) and the niche model (Williams \& Martinez 2000), to predict 16 foodweb properties. The models have 2 input parameters: the number of trophic species $(S)$ and connectance (C) of the food web being modeled. We do not report random network models (e.g. Erdös \& Rényi 1960) because food-web structure, as well as the structure of most, if not all, real-world networks is clearly not random (Watts \& Strogatz 1998, Williams \& Martinez 2000). The cascade model (Cohen et al. 1990) assigns each species a random value drawn uniformly from the interval $(0,1)$ and each species has a probability $p$ $=2 C S /(S-1)$ of consuming species with values lower than its own. The niche model (Williams \& Martinez 2000) builds on the cascade model, with each species similarly assigned a randomly drawn 'niche value' $\left(n_{\mathrm{i}}\right)$ from the interval $(1,0)$ (Fig. 1). Each species is then constrained to consume all prey species within a range of values $\left(r_{\mathrm{i}}\right)$ whose randomly chosen center $\left(c_{\mathrm{i}}\right)$ is less than the consumer's niche value. In the niche model, the placement of the feeding range relaxes the cascade model's strict feeding

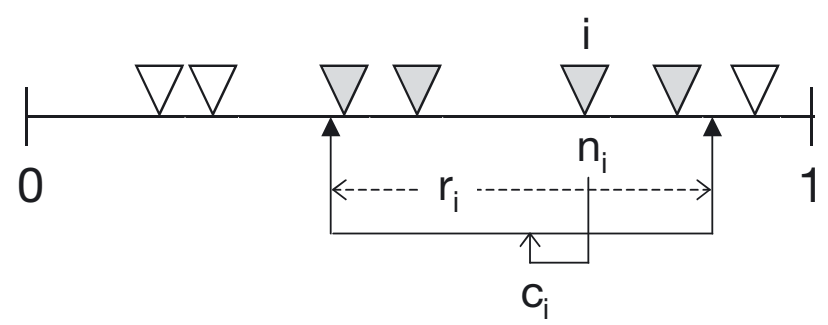

Fig. 1. Diagram of the niche model. $S$ (trophic species richness) and $C$ (connectance) are set at the observed values for the empirical web being modeled. Each of $S$ species (here $S=$ 7 , shown by inverted triangles) is assigned a 'niche value' $\left(n_{\mathrm{i}}\right)$ drawn uniformly from the interval $(0,1)$. Species $i$ consumes all species falling in a range $\left(r_{\mathrm{i}}\right)$ that is placed by uniformly by drawing the center of the range $\left(c_{\mathrm{i}}\right)$ from the interval $\left(r_{\mathrm{i}} / 2, n_{\mathrm{i}}\right)$. Thus, in this diagram, species $i$ consumes 4 species (shaded triangles) including itself. The size of $r_{\mathrm{i}}$ is assigned by using a beta function to randomly draw values from the interval $(0,1)$ whose expected value is $2 C$ and then multiplying that value by $n_{\mathrm{i}}$ to obtain a $C$ that matches the $C$ of the empirical web being modeled 
hierarchy by allowing up to half a consumer's range to include species with higher niche values than the consumer, thus allowing looping and cannibalism. Also, the consumer must feed on all species that fall within its feeding range, a contiguity that is absent from the cascade model. For each marine food web, we used Monte Carlo simulations to generate 1000 cascade and niche model webs with the same $S$ and $C$ as the empirical web, allowing calculation of a model mean and standard deviation for each of the 18 network properties. If the normalized error (raw error divided by model SD) between the empirical property and the mean model value for that property falls with \pm 2 model $S D$, the model is considered to be a good fit to the empirical data (Williams \& Martinez 2000). Due to the relatively high diversity and connectance of the NE US Shelf web, and the high diversity of the large Caribbean Reef web, the computational time required to run the models for the 3 food-chain-length related measures was prohibitive. For those webs, model results for the other 13 properties are reported.

In an extinction analysis, we simulated species loss in the Benguela, small Caribbean Reef, and NE US Shelf food webs by sequentially removing species selected by 1 of 3 criteria: (1) the most-connected species; (2) randomly chosen species (1000 random removal sequences initiated for each web); and (3) the least-connected species (Dunne et al. 2002a). We did not analyze the large version of the Caribbean Reef web due to its extreme bias discussed above. A species' connectedness is the sum of both its predator and prey links. When the most- and least-connected species are targeted for 'primary extinctions,' we protect basal species because removing such highly aggregated species (e.g. 'phytoplankton') in marine food webs has obviously large and not particularly informative consequences. 'Secondary extinctions' result when a consumer species loses all of its prey items or when a cannibalistic species loses all of its prey items except its own species. The most- or leastconnected species in a web was determined at each step of the simulation, i.e. after the web had reconfigured after the previous removal and any resulting secondary extinctions. The structural 'robustness' of each food web to the 3 types of species loss was calculated as the fraction of species that had to be removed in order to result in total species loss (i.e. primary species removals plus secondary extinctions) of $\geq 50 \%$ of the species in the original web. Maximum robustness $(0.50)$ occurs when no secondary extinctions follow primary extinction of $50 \%$ of the species and minimum robustness $(1 / S)$ occurs when the first primary extinction leads to $50 \%$ total loss of species (Dunne et al. 2002a).

\section{RESULTS AND DISCUSSION}

\section{Direct comparisons of food webs}

Direct comparison of some common food-web properties suggests that the 3 marine ecosystems, as represented by the Benguela, small Caribbean Reef, and NE US Shelf food webs, have strong structural similarities (Tables $1 \& 2$ ). The trophic species version of these 3 webs have similar numbers of top ( $T, 0$ to $4 \%$ ), intermediate ( $I, 93$ to $94 \%)$, and basal taxa ( $B, 3$ to $7 \%$ ), as well as omnivores (Omn, 76 to $86 \%$ ). Mean shortest path length among all pairs of taxa is nearly identical ( 1.6 links) and $C$ ranges from 0.22 to 0.24 . Consistent with current food-web theory (e.g. Martinez 1992), L/S and chain length vary strongly among the marine webs, increasing with $S$. Thus, Benguela, with 29 taxa, has the smallest values $(L / S=7.0$, mean chain length $=$ 6.4) and NE US Shelf, with 79 taxa, has the largest values $(L / S=17.8$, mean chain length $=15.3)$.

How do marine food webs compare with food webs from other types of ecosystems? Direct comparisons of food-web properties across and often within ecosystem types reveal a wide range of values (Table 2). Since many food-web properties are scale-dependent, in that they depend on the diversity and complexity of a system (e.g. Martinez 1994, Williams \& Martinez 2000, Dunne et al. 2002b), this variation is neither surprising nor strongly suggestive of fundamental differences among food webs. However, given that caveat, marine food webs have levels of $I$ (93 to $94 \%$ ), Omn (76 to $86 \%$ ), and Can (24 to $42 \%$ ) taxa that are on the high end of the range of values across all of the food webs, as suggested by previous studies (Cohen 1994, Link 2002). Levels of $I, O m n$, and Can in the marine webs are comparable to 2 small webs (Coachella Valley and Skipwith Pond) also noted by Link (2002) for the NE US Shelf web. The high levels of I, Omn, and Can in these 2 non-marine webs appear due to high aggregation of taxa in the Coachella Valley web and the predominance of generalist (wide-diet) insect species in the Skipwith Pond web. While marine food webs define the high end of the range of Omn, there are a number of food webs besides Coachella Valley and Skipwith Pond that show levels of omnivory greater than 55 \% (St. Martin Island, El Verde Rainforest, Mirror Lake, Lake Tahoe, St. Mark's Estuary, and Ythan Estuary with parasites).

Whether high I, Omn, and Can in marine webs result from methodological problems with marine data (e.g. uneven resolution with a bias towards omnivorous fishes), from methodological problems with non-marine data (e.g. the tendency to overlook cannibalistic interactions), or from fundamental differences of marine versus non-marine ecosystems (e.g. 
Table 2. Some commonly reported structural food-web properties for 19 food webs from a variety of ecosystem types. Taxa $=$ number of taxa from original food web, $S=$ number of trophic species, $C=$ connectance $\left(L / S^{2}\right), L / S=$ links per species, ChLen $=$ mean food chain length, TL = mean trophic level, Path = characteristic path length, $T=\%$ top species, $I=\%$ intermediate species, $B=\%$ basal species, Can $=\%$ cannibalistic species, Omn $=\%$ omnivorous species. See Dunne et al. (2002a) for more detailed descriptions of the non-marine datasets. Food webs are arranged from least to most trophic species $(S)$ within a given ecosystem type

\begin{tabular}{|c|c|c|c|c|c|c|c|c|c|c|c|c|c|}
\hline & Taxa & $S$ & $C$ & $L / S$ & ChLen & $\mathrm{TL}$ & Path & $T$ & $I$ & $B$ & Can & Omn & Source \\
\hline \multicolumn{14}{|l|}{ Terrestrial } \\
\hline Coachella Valley ${ }^{a}$ & 30 & 29 & 0.31 & 9.0 & 6.7 & 3.0 & 1.4 & 0 & 90 & 10 & 66 & 76 & Polis (1991) \\
\hline St. Martin Island ${ }^{\mathrm{a}}$ & 44 & 42 & 0.12 & 4.9 & 5.2 & 2.4 & 1.9 & 17 & 69 & 14 & 0 & 60 & $\begin{array}{l}\text { Goldwasser \& Reagan } \\
\text { (1996) }\end{array}$ \\
\hline UK Grassland & 75 & 61 & 0.03 & 1.6 & 3.2 & 2.6 & 3.7 & 31 & 56 & 13 & 0 & 21 & Memmott et al. (2000) \\
\hline El Verde & 156 & 155 & 0.06 & 9.7 & 8.4 & 2.5 & 2.3 & 13 & 69 & 18 & 1 & 57 & Waide \& Reagan (1996) \\
\hline \multicolumn{14}{|l|}{ Lake/Pond } \\
\hline Skipwith Pond ${ }^{a}$ & 35 & 25 & 0.32 & 7.9 & 6.2 & 2.7 & 1.3 & 4 & 92 & 4 & 32 & 60 & Warren (1998) \\
\hline Bridge Brook Lake $^{a}$ & 75 & 25 & 0.17 & 4.3 & 4.0 & 2.0 & 1.9 & 0 & 68 & 32 & 12 & 40 & Havens (1992) \\
\hline Little Rock Lake ${ }^{\mathrm{a}}$ & 182 & 92 & 0.12 & 10.8 & 7.3 & 2.4 & 1.9 & 1 & 86 & 13 & 14 & 38 & Martinez (1991) \\
\hline Mirror Lake & 586 & 172 & 0.15 & 25.1 & 9.1 & 2.1 & 1.8 & 1 & 74 & 25 & 17 & 59 & Martinez (unpubl. data) \\
\hline Lake Tahoe & 800 & 172 & 0.13 & 22.6 & 10.7 & 2.1 & 1.9 & 9 & 66 & 28 & 17 & 58 & Martinez (unpubl. data) \\
\hline \multicolumn{14}{|l|}{ Stream } \\
\hline Canton Creek & 108 & 102 & 0.07 & 6.8 & 3.2 & 1.5 & 2.3 & 25 & 22 & 53 & 1 & 8 & Townsend et al. (1998) \\
\hline Stony Stream & 112 & 109 & 0.07 & 7.6 & 3.1 & 1.5 & 2.3 & 17 & 27 & 56 & 2 & 10 & Townsend et al. (1998) \\
\hline \multicolumn{14}{|l|}{ Estuary } \\
\hline Chesapeake Bay ${ }^{\mathrm{a}}$ & 33 & 31 & 0.07 & 2.2 & 4.0 & 2.4 & 2.7 & 32 & 52 & 16 & 3 & 52 & Baird \& Ulanowicz (1989) \\
\hline St. Mark's Estuary & 48 & 48 & 0.10 & 4.6 & 6.6 & 2.5 & 2.0 & 17 & 69 & 12 & 6 & 71 & $\begin{array}{l}\text { Christian \& Luczkovich } \\
\text { (1999) }\end{array}$ \\
\hline Ythan Estuary ${ }^{\mathrm{a}}$ & 92 & 83 & 0.06 & 4.8 & 5.9 & 2.6 & 2.2 & 37 & 54 & 9 & 4 & 54 & Hall \& Raffaelli (1991) \\
\hline $\begin{array}{l}\text { Ythan Estuary } \\
\text { with parasites }\end{array}$ & 134 & 124 & 0.04 & 4.7 & 6.3 & 2.9 & 2.4 & 40 & 56 & 4 & 3 & 62 & Huxham et al. (1996) \\
\hline \multicolumn{14}{|l|}{ Marine } \\
\hline Benguela & 29 & 29 & 0.24 & 7.0 & 6.4 & 3.2 & 1.6 & 0 & 93 & 7 & 24 & 76 & Yodzis (1998) \\
\hline $\begin{array}{l}\text { Caribbean Reef, } \\
\text { small }\end{array}$ & 50 & 50 & 0.22 & 11.1 & 9.8 & 2.9 & 1.6 & 0 & 94 & 6 & 42 & 86 & Opitz (1996) \\
\hline NE US Shelf & 81 & 79 & 0.22 & 17.8 & 15.3 & 3.1 & 1.6 & 4 & 94 & 3 & 32 & 78 & Link (2002) \\
\hline $\begin{array}{l}\text { Caribbean Reef, } \\
\text { large }\end{array}$ & 249 & 245 & 0.05 & 13.8 & 10.5 & 3.1 & 1.9 & 0 & 98 & 2 & 4 & 87 & Opitz (1996) \\
\hline
\end{tabular}

unusually widespread generality in marine systems related to gape size and filter feeding), continues to be an open question that requires more detailed and evenly resolved data to settle (Cohen et al. 1993). The excessively low percentage of basal taxa in these marine food webs compared to other systems is clearly an artifact of poor resolution of primary producers and consumer links to them. Better resolution at the basal level would tend to mitigate, but probably not erase, current high levels of $I, O m n$, and Can in marine food webs.

As also discussed by Link (2002) for the NE US Shelf web, and Cohen (1994) for a set of 11 highly aggregated low-diversity marine food webs, the 3 marine webs examined here have high $L / S$ and mean chain lengths compared to most other food webs from other ecosystems, particularly the small Caribbean Reef and NE US Shelf webs (Table 2). However, Lake Tahoe and
Mirror Lake have greater $L / S$, and there are a number of other food webs (Coachella Valley, El Verde Rainforest, Skipwith Pond, and Little Rock Lake) with comparable $L / S$. The NE US Shelf displays the longest mean chain length (15.3), but the small Caribbean Reef, Lake Tahoe, and Mirror Lake food webs display comparable mean chain lengths of $\sim 9$ to 11 . Six other webs fall close to (Coachella Valley, Skipwith Pond, St. Mark's Estuary, Ythan Estuary with parasites) or greater than (El Verde Rainforest, Little Rock Lake) Benguela's mean chain length of 6.4. In addition to the known diversity dependence of these measures, Cohen (1994) extensively discusses a variety of other biological and methodological reasons that marine webs might display high $L / S$ and chain lengths relative to non-marine webs.

The 3 marine food webs have relatively high $C$ of 0.22 to 0.24 , which is within the previously observed 
Table 3. Comparison of 16 empirically observed structural food-web properties with niche model means in parentheses for 3 marine food webs. Empirical values whose normalized error falls within \pm 2 model $S D$, demonstrating a good fit between the model and empirical data, are shown in bold. Niche model food-chain properties were not calculated for the NE US Shelf and the large Caribbean Reef food webs due to the excessive computing time required. Herb $=\%$ herbivores plus detritivores, Loop $=\%$ species in loops, GenSD = generality standard deviation, $\operatorname{VulSD}=$ vulnerability standard deviation, $\mathrm{MaxSim}=$ mean maximum similarity, $C h S D=$ food chain length standard deviation, ChNum $=\log$ food chain number, Clust $=$ clustering coefficient. See Table 2 for other definitions

\begin{tabular}{|c|c|c|c|c|}
\hline & Benguela & $\begin{array}{l}\text { Caribbean } \\
\text { Reef, small }\end{array}$ & $\begin{array}{l}\text { Caribbean } \\
\text { Reef, large }\end{array}$ & $\begin{array}{l}\text { NE US } \\
\text { Shelf }\end{array}$ \\
\hline$T$ & $\mathbf{0}(5)$ & $\mathbf{0}(3)$ & $\mathbf{0}(4)$ & $4(2)$ \\
\hline$I$ & $93(83)$ & $94(88)$ & $98(86)$ & $94(92)$ \\
\hline$B$ & $7(12)$ & $6(9)$ & $2(10)$ & $3(6)$ \\
\hline$H_{e r b}{ }^{\mathrm{a}}$ & $7(5)$ & $6(4)$ & $4(4)$ & $19(2)$ \\
\hline Can & $24(31)$ & 42 (29) & $4(6)$ & $32(30)$ \\
\hline Omn & $76(78)$ & 86 (83) & $87(48)$ & 78 (88) \\
\hline Loop & $41(56)$ & $68(68)$ & 73 (35) & $67(78)$ \\
\hline GenSD & $\mathbf{0 . 8 8}(0.91)$ & $\mathbf{0 . 9 0}(0.93)$ & $1.92(1.20)$ & $\mathbf{0 . 9 0}(0.92)$ \\
\hline VulSD & $0.73(0.55)$ & $\mathbf{0 . 6 1}(0.55)$ & $1.18(0.59)$ & $0.73(0.53)$ \\
\hline MaxSim & $\mathbf{0 . 6 6}(0.70)$ & $0.58(0.74)$ & $0.61(0.68)$ & $0.70(0.77)$ \\
\hline$T L^{\mathrm{a}}$ & $3.2(3.2)$ & $2.9(3.5)$ & $3.1(3.0)$ & $3.1(3.8)$ \\
\hline ChLen & $6.4(7.5)$ & $\mathbf{9 . 8}(10.3)$ & - & - \\
\hline$C h S D$ & $1.5(1.6)$ & 2.1 (1.9) & - & - \\
\hline ChNum & $3.8(4.4)$ & $6.3(6.5)$ & - & - \\
\hline Path $^{\mathrm{a}}$ & $1.6(1.6)$ & $1.6(1.6)$ & $1.9(2.2)$ & $1.6(1.6)$ \\
\hline Clust $^{\mathrm{a}}$ & $\mathbf{0 . 3 0}(0.36)$ & $\mathbf{0 . 3 6}(0.34)$ & $0.16(0.10)$ & $\mathbf{0 . 3 1}(0.34)$ \\
\hline
\end{tabular}

confounds direct assessment of the similarity, or dissimilarity, of food-web structure. Using direct comparisons makes it difficult to gauge the significance of differences, especially when the available datasets are few and highly variable both ecologically and methodologically. Instead, an approach that uses simple models that incorporate variable diversity and connectance, such as the niche model (Williams \& Martinez 2000) and certain implementations (Williams \& Martinez 2000) of the cascade model (Cohen et al. 1990), provides a more robust way to evaluate empirical food-web structure.

The cascade model fails to accurately predict marine food-web structure. Across all 3 higher-quality marine webs, the cascade model provides a good fit for only $33 \%$ of the properties examined: 5 of 16 properties for Benguela, 6 of 16 for the small Caribbean Reef, and 4 of 13 for the NE US Shelf (results not shown). The cascade model disallows looping and cannibalism, which occurs in all 3 food webs. The cascade model also system-

range of 0.03 to 0.3 (Table 2, Dunne et al. 2002a). We do not follow Link's (2002) use of 'connectivity,' also known as 'interactive connectance' $(L /[S(S-1) / 2])$. The denominator represents less than $1 / 2$ of the full predation matrix and renders cannibalism and mutual predation links impossible, while the numerator includes such links, leading to double counting of links and exaggerated connectance (Martinez 1991). Instead, we use 'directed connectance' $\left(C=L / S^{2}\right)$ (Martinez 1991, 1992) because it avoids these problems by forcing the numerator to account for mutual predation and cannibalism links (Polis 1991), and allowing for the possibility for any species to feed on any species in a web (including the potential for plants to parasitize other plants). Thus, the assertion that an order-ofmagnitude higher connectivity in the NE US Shelf food web indicates dramatic differences between marine and other types of ecosystems (Link 2002) appears incorrect. Instead, the marine food webs examined here display connectance above the mean but within the range seen in non-marine food webs.

\section{Comparisons using the food-web models}

As discussed in the 'Introduction', the dependence of many food-web properties on diversity and complexity atically underestimates 4 other properties across all 3 webs: GenSD, MaxSim, Path, and Clust. For Benguela and the small Caribbean Reef, the 2 food webs for which computationally intensive chain length metrics could be modeled, the cascade model overestimated all 3 chain length properties. These results are very similar to an analysis of network structure of 2 lake, 1 pond, 2 estuary, and 2 terrestrial food webs (Williams \& Martinez 2000, Table 2), where the overall success rate of the cascade model was $27 \%$.

In contrast, the niche model more closely predicts the structure of the 3 marine food webs. Of 16 niche model means, all 16 are within 2 model SD of the empirical values for Benguela, with a good fit for 14 of $16(88 \%)$ properties for the small Caribbean Reef web (Table 3). The niche model underestimates Can and overestimates MaxSim in the small Caribbean Reef web. The niche model predicts the structure of the NE US Shelf web less well than the other 2 marine food webs, but still much better than the cascade model. Nine of $13(69 \%)$ properties are well predicted, with VulSD and Herb underestimated and Omn and MaxSim overestimated by the niche model. The drastic underestimation of Herb may be connected to the hyper-aggregation of basal groups, which represent only $3 \%$ of taxa in the NE US Shelf web; very low compared to most other food webs 
(Table 2). However, it may also reflect a limitation of the niche model, which tends to underestimate Herb for most food webs (data not shown).

While the niche model appears able to identify fundamental food-web structure that is fairly robust to some bias, variability, and aggregation in the data, it is sensitive to systematic bias. For example, in all 3 marine webs the niche model underestimates VulSD. This underestimation may occur due to methodological bias in these marine food webs towards inclusion of high trophic level taxa (i.e. fishes) without identifying their full complements of predators or parasites (Williams \& Martinez 2000). The niche model is also sensitive to extreme bias in particular webs. Consider the large Caribbean Reef food web, with $84 \%$ fish taxa and only 0.05 connectance. The niche model does a poor job of predicting its network structure as illustrated by a good fit to only 6 of 13 properties (46\%) (Table 3). This is better than the cascade model, which successfully predicts only 3 of 13 properties (23\%), but far worse than the success of the niche model for the other 3 marine webs. The niche model's failure to fit the structure of the large Caribbean Reef food web echoes the relatively poor fit of the niche model ( 7 of 12 properties) to the data for the Ythan Estuary food web (Hall \& Raffaelli 1991), which appears related to overrepresentation of top bird species (Williams \& Martinez 2000).

For the 3 more evenly resolved marine food webs, the average $87 \%$ predictive success rate of the niche model is similar to its $79 \%$ success rate across 7 nonmarine food webs (Williams \& Martinez 2000). While the 16 properties currently examined are not completely independent, and the degree to which they covary has yet to be assessed, there is clearly a strong difference in how accurately the cascade and niche models characterize the topology of food webs. The similarly high level of the niche model's predictive success for marine, estuary, freshwater, and terrestrial food webs suggests that marine and non-marine food webs have remarkably similar network structure, once diversity and connectance levels are incorporated. However, because the niche model assumes a particular level of connectance, it does not explain why marine food webs appear to have relatively high trophic interaction richness compared to other webs. Both of these aspects of marine food-web structure, i.e. its niche-model similarity to the structure of other food webs, as well as its differences in connectance from other webs, must be considered provisional until more highly and evenly resolved data for lower-trophic-level taxa and interactions are incorporated into marine food-web datasets, and until a greater variety and higher quality of all types of food webs are compiled and analyzed.

\section{Structural robustness of marine food webs to species loss}

We systematically removed species from the 3 higher-quality marine food webs in biodiversity-loss simulations. The secondary extinctions resulting from the loss of all prey items varied both among webs and among types of species removed (Fig. 2). Very few secondary extinctions occurred as a result of removing taxa with very few connections, whereas removing

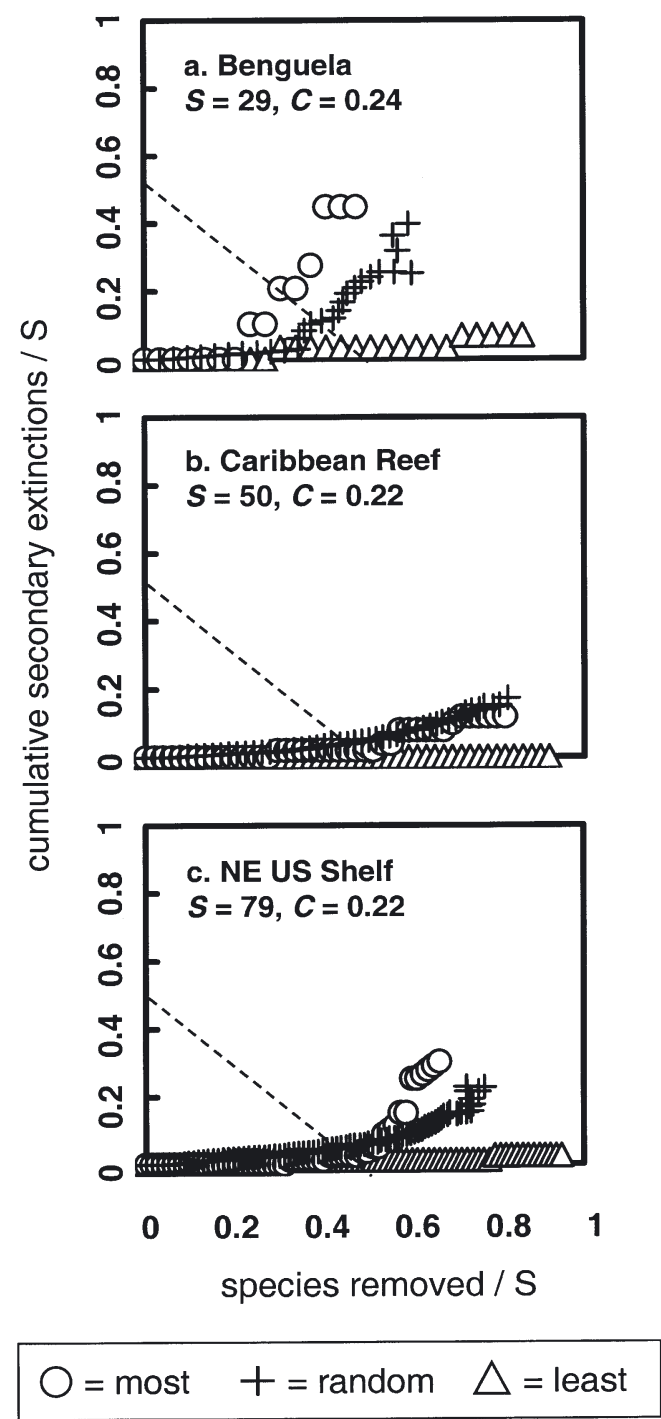

Fig. 2. Secondary extinctions resulting from 3 types of primary species removal in 3 marine food webs. $95 \%$ error bars for random species removals fall within the size of the symbols and are not shown. Dashed line shows the points at which there is $\geq 50 \%$ total species loss (primary species removals plus secondary extinctions) in a food web for each type of species removal. Proportion of species that must be removed to reach that point is referred to as 'structural robustness'. $S$ : species richness; $C$ : connectance (links per species $^{2}, L / S^{2}$ ) 
random taxa and the most-connected taxa did result in additional species loss after $25 \%$ of the species in the original webs were removed. Benguela showed the most dramatic effects and differences among the 3 targeting strategies, where removal of $\sim 30 \%$ of mostconnected, random, and least-connected taxa resulted in $\sim 20,11$, and $3 \%$ of the taxa undergoing secondary extinctions, respectively. In general, the small Caribbean Reef and NE US Shelf web displayed very high robustness to species removals, with $>50 \%$ primary removals required to induce $>5 \%$ secondary extinctions.

Marine food webs generally show high structural robustness to species loss compared to other food webs analyzed previously (Solé \& Montoya 2001, Dunne et al. 2002a), which appears related to their relatively high connectance (Fig. 3, Dunne et al. 2002a). When most-connected or random species are targeted, structural robustness increases significantly with increasing connectance, and saturates at 0.50 , which is the point at which there are no secondary extinctions as a result of $50 \%$ primary removals (Fig. 3). Targeting leastconnected species was not significantly related to $C$, partially because of the low level of secondary extinctions that resulted (results not shown). Benguela is an outlier compared to the other 2 marine and 16 nonmarine webs represented, with lower robustness than expected for both random and most-connected species

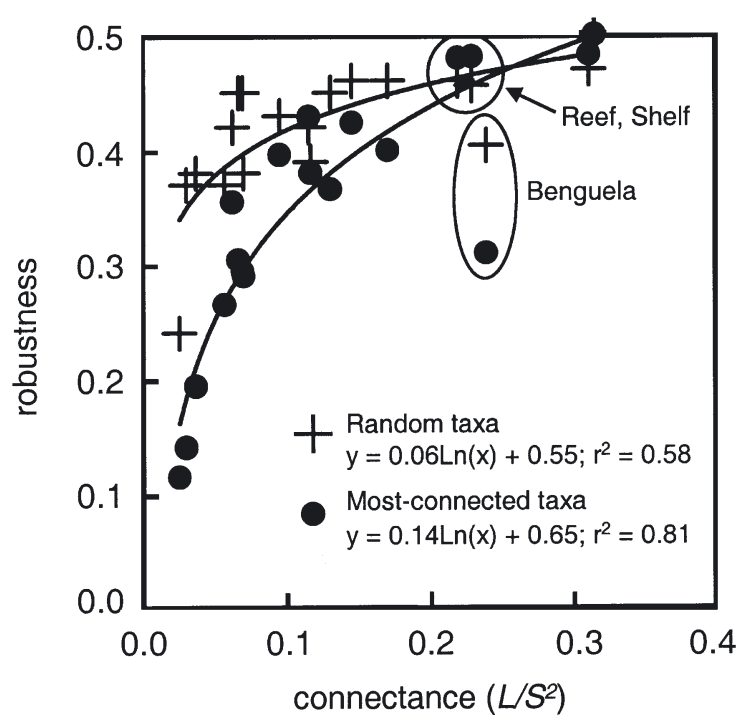

Fig. 3. Structural robustness (the proportion of primary species removals required to induce a total loss of $\geq 50 \%$ of species) as a function of connectance $\left(L / S^{2}\right)$ in 16 food webs from Dunne et al. (2002a) plus 3 recent marine food webs (circled data points). Data shown for loss of most-connected taxa are for deletion sequences where basal taxa are protected from removal. Maximum robustness value $=0.50$ (i.e. no secondary extinctions) removals. Structural robustness did not vary significantly with $S$, regardless of type of species loss (linear regression data not shown, see Dunne et al. 2002a).

Why is Benguela an outlier? The first 10 most- and least-connected taxa removed in the simulations are shown for each food web in Table 4 . In the Benguela food web, there are highly connected taxa such as macro- and mesozooplankton that are at fairly low trophic levels in the food web, unlike in the small Caribbean Reef and NE US Shelf webs. This may suggest that from a structural perspective, removing highly connected lower trophic level taxa is even more destabilizing than removing highly connected upper trophic level taxa.

\section{CONCLUSIONS}

Previous examinations of marine food-web structure concluded that marine food webs are fundamentally different from other kinds of food webs, based on their high $L / S$, connectivity, and chain lengths; differences which may relate in part to high levels of omnivorous, generalist organisms in marine habitats (Cohen 1994, Link 2002). The current study corroborates aspects of the previous studies. Direct comparisons of measures such as $L / S$, connectance, omnivory, and cannibalism suggest that marine food webs: (1) tend to look similar to each other; and (2) have high values for those properties compared to estuary, freshwater, and terrestrial food webs. Marine webs thus appear to have relatively high trophic interaction richness. However, in most cases marine food webs do fall within previously reported ranges of observed food-web properties from non-marine systems, and the least diverse marine web (Benguela) has values that are often quite similar to those of many nonmarine food webs. More evenly and highly resolved data from marine and non-marine systems will help to decide whether current patterns are artifacts or whether they reflect more significant similarities or differences among and within different types of ecosystems.

Even given possible differences in food-web properties between marine and other ecosystems, it does not appear that 'food web theory needs to be modified to accommodate observations from marine ecosystems' (Link 2002). Instead, our study shows that when species richness and connectance are taken into account, observations from marine food webs corroborate current theory concerning the topology of food webs. In particular, the niche model very accurately predicts the structure of marine food webs, particularly the Benguela and small Caribbean Reef webs, as it does for other types of food webs (Williams \& Martinez 2000, 
Table 4. First 10 taxa eliminated from 3 marine food webs when targeting the most and least connected taxa, with basal taxa protected from removal. Qualifiers in parentheses refer to body size classes (int. = intermediate). Names are drawn from the original studies

\begin{tabular}{|c|c|c|c|}
\hline & Benguela & Caribbean Reef, small & NE US Shelf \\
\hline \multicolumn{4}{|c|}{ Most connected taxa } \\
\hline 1 & Sharks & Sharks/rays (large) & Cod \\
\hline 2 & Hakes & Carnivorous reef fish \#3 (int.) & Red hake \\
\hline 3 & Squid & $\begin{array}{l}\text { Shrimps/hermit crabs/ } \\
\text { stomatopods }\end{array}$ & Spotted hake \\
\hline 4 & Birds & Carnivorous reef fish \#2 (int.) & White hake \\
\hline 5 & $\begin{array}{l}\text { Macrozoo- } \\
\text { plankton }\end{array}$ & Carnivorous reef fish \#1 (small) & Silver hake \\
\hline 6 & $\begin{array}{l}\text { Other } \\
\quad \text { groundfish }\end{array}$ & Omnivorous reef fish \#1 (small) & Little skate \\
\hline 7 & $\begin{array}{l}\text { Mesozoo- } \\
\text { plankton }\end{array}$ & Crabs & Spiny dogfish \\
\hline 8 & Anchovy & Gastropods & Goosefish \\
\hline 9 & $\begin{array}{l}\text { Benthic } \\
\text { carnivores }\end{array}$ & Carnivorous reef fish (large) & Winter skate \\
\hline 10 & Goby & Omnivorous reef fish \#2 (small) & Other decapods \\
\hline \multicolumn{4}{|c|}{ Least connected taxa } \\
\hline 1 & Bacteria & Kyphosidae & Snails \\
\hline 2 & $\begin{array}{l}\text { Zooplankton, } \\
\text { gelatinous }\end{array}$ & Gobiidae (small) & Tunicates \\
\hline 3 & $\begin{array}{l}\text { Microzoo- } \\
\text { plankton }\end{array}$ & Groupers (large) & Billfish \\
\hline 4 & Lightfish & Hemiramphidae & Birds \\
\hline 5 & Other pelagics & Scaridae (large) & Seals \\
\hline 6 & Yellowtail & Scaridae (int.) & Pteropods \\
\hline 7 & Geelbek & Scaridae (int.) & Sponges \\
\hline 8 & $\begin{array}{c}\text { Whales and } \\
\text { dolphins }\end{array}$ & Sea turtles & Sea cucumbers \\
\hline 9 & $\begin{array}{l}\text { Benthic } \\
\text { carnivores }\end{array}$ & Lobsters & Baleen whales \\
\hline 10 & Tunas & Jacks (large) & Calanus sp. \\
\hline
\end{tabular}

induce higher levels of secondary extinctions than loss of random taxa (Albert et al. 2000, Solé \& Montoya 2001, Dunne et al. 2002a). This suggests that marine systems, if their relatively high connectance is not an artifact of methodology, will have greater inherent structural robustness than other ecosystems. However, other structural effects can come into play. First, extinctions in marine systems do not appear random, particularly with regard to anthropogenic effects over the last several centuries. Humans tend to selectively and intensively take marine taxa at high trophic levels (Pauly et al. 1998, Jackson et al. 2001), which are often the taxa that are highly connected (Table 4). Also, the mean shortest path length between pairs of taxa within marine webs is low (1.6 links) compared to other types of food webs, which have values ranging from 1.3 to 3.7 (Table 2, Dunne et al. 2002b). This suggests that most species in marine food webs are potentially very close 'neighbors,' and that negative effects can spread rapidly and widely throughout the food web (Williams et al. 2002). However, the rich network of interactions quantified by high connectance and low path length may also suggest that strong effects can rapidly disperse throughout Williams et al. 2002). This suggests that the network structure of food webs is a general property of ecosystems. It also suggests that the simple rules of the niche model, which specify a relaxed feeding hierarchy that allows cannibalism and looping as well as a contiguity of feeding within a niche, may point to common ecological, evolutionary, and thermodynamic mechanisms shaping the complex network structure of food webs from all types of ecosystems examined thus far (Williams \& Martinez 2000).

Such aspects of marine food webs are particularly interesting because of the intense economic fishing pressure, overexploitation, and collapse of many coastal and shelf marine fisheries (e.g. Boreman et al. 1997, Jackson et al. 2001). What can network structure tell us about the potential robustness of food webs to biodiversity loss? Current and previous results (Dunne et al. 2002a) suggest that high connectance communities will tend to be more robust to species loss than low connectance communities, and that the loss of highly connected taxa will tend to marine food webs, thus decreasing the overall impact of any particular fluctuation (Link 2002).

This analysis, like other structural food-web studies, does not incorporate the dynamics of interacting taxa. Useful predictions of ecosystem response to perturbations such as population or biomass fluctuations, changes in size and age class distributions, and species losses or introductions require models that integrate ecologically plausible diversity and network structure with non-linear population dynamics of species (Yodzis 1998, 2000, Williams \& Martinez in press). Studies that ignore or drastically simplify structure (e.g. approaches focused on 2 species, food chains, or small modules) or dynamics (e.g. mass-balance, Lotka-Volterra, and topological approaches) may provide unreliable approximations of the structurally complex, nonequilibrium, nonlinear, diffuse dynamics that likely characterize most ecosystems (Yodzis 2000). Marine food webs, particularly those strongly impacted by humans, may be the ideal test case for developing and applying integrated structure and dynamics models, as explored 
in the Benguela food web (Yodzis 1998, 2000). The desperate need for more sophisticated interdisciplinary ecological and economic models to assist fisheries and marine reserve policy formulation is clear (Micheli et al. 2001). An improved understanding of marine food-web structure, which explicitly focuses on the complex network of interactions in which exploited taxa are embedded, should be an important part of future marine ecosystem research and policy.

Acknowledgements. This work was supported by NSF grants DEB/DBI-0074521 (J.A.D), DEB-0083929 (N.D.M), DBI0234980 (N.D.M., J.A.D., R.J.W.), and DBI-9904777 (R.J.W.). We thank J. Link for sharing the NE US Shelf data, and C. Melian for providing the large Caribbean Reef dataset. We are grateful to D. Stouffer for pointing out errors in the data. Please contact J.A.D. for data referred to in this paper.

\section{LITERATURE CITED}

Albert R, Barabási AL (2002) Statistical mechanics of complex networks. Rev Mod Phys 74:47-97

Albert R, Jeong H, Barabási AL (2000) Error and attack tolerance of complex networks. Nature 406:378-382

Baird D, Ulanowicz RE (1989) The seasonal dynamics of the Chesapeake Bay ecosystem. Ecol Monogr 59:329-364

Boreman J, Nakashima BS, Wilson HA, Kendall RL (1997) Northwest Atlantic groundfish: perspectives on a fishery collapse. American Fisheries Society, Bethesda, MD

Briand F, Cohen JE (1984) Community food webs have scaleinvariant structure. Nature 398:330-334

Camacho J, Guimerà R, Amaral LAN (2002a) Analytical solution of a model for complex food webs. Phys Rev Lett E 65: 030901

Camacho J, Guimerà R, Amaral LAN (2002b) Robust patterns in food web structure. Phys Rev Lett 88:228102

Christensen V, Pauly D (1992) ECOPATH II: a software for balancing steady-state ecosystem models and calculating network characteristics. Ecol Model 61:169-185

Christian RR, Luczkovich JJ (1999) Organizing and understanding a winter's seagrass foodweb network through effective trophic levels. Ecol Model 117:99-124

Cohen JE (1994) Marine and continental food webs: three paradoxes? Phil Trans R Soc Lond B 343:57-69

Cohen JE, Briand F, Newman CM (1990) Community food webs: data and theory. Springer-Verlag, New York

Cohen JE, Beaver RA, Cousins SH, De Angelis DL and 20 others (1993) Improving food webs. Ecology 74:252-258

Dunbar MJ (1954) Arctic and subarctic marine ecology: immediate problems. Arctic 7:213-228

Dunne JA, Williams RJ, Martinez ND (2002a) Network structure and biodiversity loss in food webs: robustness increases with connectance. Ecol Lett 5:558-567

Dunne JA, Williams RJ, Martinez ND (2002b) Food-web structure and network theory: the role of connectance and size. Proc Natl Acad Sci USA 99:12917-12922

Erdös P, Rényi A (1960) On the evolution of random graphs. Publ Math Inst Hung Acad Sci 5:17-61

Fussman GF, Heber G (2002) Food web complexity and chaotic population dynamics. Ecol Lett 5:394-401

Goldwasser L, Roughgarden JA (1993) Construction of a large Caribbean food web. Ecology 74:1216-1233

Hall SJ, Raffaelli D (1991) Food-web patterns: lessons from a species-rich web. J Anim Ecol 60:823-842
Havens K (1992) Scale and structure in natural food webs. Science 257:1107-1109

Huxham M, Beany S, Raffaelli D (1996) Do parasites reduce the chances of triangulation in a real food web? Oikos 76: $284-300$

Jackson JB, Kirby MX, Berger WH, Bjorndal KA and 15 others (2001) Historical overfishing and the recent collapse of coastal ecosystems. Science 293:629-638

Krause AE, Frank KA, Mason DM, Ulanowicz RE, Taylor WW (2003) Compartments revealed in food-web structure. Nature 426:282-285

Link J (2002) Does food web theory work for marine ecosystems? Mar Ecol Prog Ser 230:1-9

Martinez ND (1991) Artifacts or attributes? Effects of resolution on the Little Rock Lake food web. Ecol Monogr 61: 367-392

Martinez ND (1992) Constant connectance in community food webs. Am Nat 139:1208-1218

Martinez ND (1993) Effect of scale on food web structure. Science 260:242-243

Martinez ND (1994) Scale-dependent constraints on foodweb structure. Am Nat 144:935-953

Martinez ND, Hawkins BA, Dawah HA, Feifarek BP (1999) Effects of sampling effort on characterization of food-web structure. Ecology 80:1044-1055

Memmott J, Martinez ND, Cohen JE (2000) Predators, parasitoids and pathogens: species richness, trophic generality and body sizes in a natural food web. J Anim Ecol 69:1-15

Micheli F, Polis GA, Boersma PD, Hixon MA, Norse EA, Snelgrove PVR, Soulé ME (2001) Human alteration of food webs: research priorities for conservation and management. In: Soulé ME, Orians GH (eds) Conservation biology: research priorities for the next decade, 2nd edn. Island Press, Washington, DC, p 31, 58

Montoya JM, Solé RV (2002) Small world patterns in food webs. J Theor Biol 214:405-412

Neutel AM, Heesterbeek JAP, de Ruiter PC (2002) Stability in real food webs: weak links in long loops. Science 296: $1120-1123$

Opitz S (1996) Trophic interactions in Caribbean coral reefs. ICLARM Tech Rep 43, Manila, Philippines

Pauly D, Christensen V, Dalsgaard J, Froese R, Torres F (1998) Fishing down marine food webs. Science 279: 860-863

Pauly D, Christensen V, Guënette S, Pitcher TJ, Sumaila UR, Walters CJ, Watson R, Zeller D (2002) Toward sustainability in world fisheries. Nature 418:689-695

Polis GA (1991) Complex desert food webs: an empirical critique of food web theory. Am Nat 138:123-155

Schoener TW (1989) Food webs from the small to the large. Ecology 70:1559-1589

Solé RV, Montoya JM (2001) Complexity and fragility in ecological networks. Proc R Soc Lond B 268:2039-2045

Strogatz SH (2001) Exploring complex networks. Nature 410: $268-275$

Townsend CR, Thompson RM, McIntosh AR, Kilroy C, Edwards E, Scarsbrook MR (1998) Disturbance, resource supply, and food-web architecture in streams. Ecol Lett 1: 200-209

Waide RB, Reagan WB (eds) (1996) The food web of a tropical rainforest. University of Chicago Press, Chicago

Warren PH (1989) Spatial and temporal variation in the structure of a freshwater food web. Oikos 55:299-311

Watts DJ, Strogatz SH (1998) Collective dynamics of 'smallworld' networks. Nature 393:440-442

Williams RJ, Martinez ND (2000) Simple rules yield complex food web. Nature 404:180-183 
Williams RJ, Martinez ND (in press) Stabilization of chaotic and non-permanent food web dynamics. Eur J Phys B

Williams RJ, Martinez ND (2004) Trophic levels in complex food webs: theory and data. Am Nat 163:458-468

Williams RJ, Berlow EL, Dunne JA, Barabási AL, Martinez ND (2002) Two degrees of separation in complex food webs.

Editorial responsibility: Andrea Belgrano,

Santa Fe, New Mexico, USA
Proc Natl Acad Sci USA 99:12913-12916

Yodzis P (1998) Local trophodynamics and the interaction of marine mammals and fisheries in the Benguela ecosystem. J Anim Ecol 67:635-658

Yodzis P (2000) Diffuse effects in food webs. Ecology 81: 261-266

Submitted: April 16, 2003; Accepted: January 27, 2004 Proofs received from author(s): April 20, 2004 